\title{
Acute haemodynamic effects of pinacidil in man
}

\author{
D. P. NICHOLLS*, J. G. MURTAGH, M. E. SCOTT, P. MORTON \& R. G. SHANKS ${ }^{1}$ \\ The Cardiac Unit, Belfast City Hospital, Belfast BT97AB and ${ }^{1}$ Department of Therapeutics and Pharmacology, \\ The Queen's University of Belfast, Whitla Medical Building, Belfast BT9 7BL, Northern Ireland
}

1 The acute haemodynamic effects of i.v. pinacidil $0.2 \mathrm{mg} \mathrm{kg}^{-1}$ infused over $8 \mathrm{~min}$ were studied in 10 normotensive patients undergoing cardiac catheterisation.

2 Mean arterial pressure fell from $94 \pm 3 \mathrm{mmHg}$ (mean \pm s.e. mean) before infusion to $74 \pm 3 \mathrm{mmHg}$ at $10 \mathrm{~min}$ after commen ${ }^{-i n g}$ infusion $(P \div 0.001)$ and during this time heart rate increased from $75 \pm 4$ to $106 \pm 7$ beats $\min ^{-1}(P<0.001)$. Significant changes were recorded until the end of the observation period ( $70 \mathrm{~min}$ after commencing infusion).

3 Cardiac index increased from $3.2 \pm 0.2$ to $4.0 \pm 0.21 \mathrm{~min}^{-1} \mathrm{~m}^{-2}(P<0.001)$ and systemic vascular resistance fell from $16 \pm 1$ to $10 \pm 1$ units $(P<0.001)$ at $10 \mathrm{~min}$ after commencing infusion. By the end of the observation period, the values had returned to pre-infusion levels.

4 Only small changes in pulmonary haemodynamics were observed.

5 These results indicate that pinacidil acts as a peripheral arteriolar vasodilator, and as such may have a role in the treatment of arterial hypertension and of cardiac failure.

Keywords haemodynamics pinacidil normal man vasodilators

\section{Introduction}

Pinacidil is a potent arteriolar vasodilator, and in vitro studies indicate that this is due to a direct action on vascular smooth muscle (ArrigoniMartelli et al., 1980; Nielsen \& Arrigoni-Martelli, 1981; Mikkelsen \& Pedersen, 1982). In the anaesthetised dog, pinacidil produced an increase in regional blood flow when infused into the carotid, coronary, femoral or renal arteries, and when given intravenously at a dose of $0.2 \mathrm{mg}$ $\mathrm{kg}^{-1}$ produced a small reduction in arterial pressure and an increase in coronary and renal blood flow (Olsen \& Arrigoni-Martelli, 1983). In normal man, the oral administration of pinacidil produced small reductions in arterial pressure and an increase in heart rate (Nicholls et al., 1984, 1986). In hypertensive patients, the oral administration of pinacidil reduced arterial pressure (Kardel $e t$ al., 1981; Ward, 1984) and non-invasive haemodynamic studies have indicated that the profile of action of pinacidil is similar to that of other arteriolar vasodilator drugs (Carlsen et al., 1981).

We have studied the acute haemodynamic effects of pinacidil given intravenously to normotensive patients undergoing cardiac catheterisation.

\section{Methods}

\section{Patient selection}

Observations were made on 10 patients undergoing cardiac catheterisation for known or suspected ischaemic heart disease. The clinical details of the patients are shown in Table 1. All drug therapy except sublingual glyceryl trinitrate had been withheld for at least 3 days prior to the study. Patients were excluded from the study on the following grounds: a history of heart failure; previous adverse reaction to vasodilator drugs; systolic arterial pressure of less than $100 \mathrm{mmHg}$; resting left ventricular end diastolic pressure of $20 \mathrm{mmHg}$ or more; ejection fraction (calculated from left ventricular (LV) 
angiogram) of $40 \%$ or less; adverse haemodynamic reaction to LV angiography. Patients considered suitable were invited to take part on the day before the study after the details had been explained to them, and written consent was obtained. The protocol of the study had been approved by the Ethics Committee of the Queen's University of Belfast.

\section{Procedure}

All patients were fasting overnight and were given diazepam $10 \mathrm{mg}$ orally $1 \mathrm{~h}$ prior to study. Under local anaesthesia, catheters were introduced into the right femoral artery by the Seldinger technique, and coronary angiograms obtained. LV angiography was carried out using iopamidol $370\left(1 \mathrm{ml} \mathrm{kg}^{-1}\right)$ as contrast. A flowdirected triple-lumen catheter (Edwards Laboratories) was then introduced into the right femoral vein and advanced to a pulmonary artery. After simultaneous recording of the pulmonary wedged pressure and the LV end diastolic pressure, the LV catheter was withdrawn into the descending aorta to measure systemic arterial pressure. All pressures were measured with reference to a point $5 \mathrm{~cm}$ below the sternal angle in the supine position, and recorded with an electrocardiogram on a Mingograf 804 recorder (Siemens). The haemodynamic data were fed into a Siemens SICOR computer and mean pressures calculated by integration of the area under the time/pressure curve.

At each observation time, the following measurements were made: pulmonary wedged pressure, pulmonary arterial pressure, systemic arterial pressure, heart rate and thermodilution cardiac output (mean of three measurements). From the above data, the following parameters were derived: cardiac index = cardiac output/ body surface area from nomogram; systemic vascular resistance $=$ (mean systemic arterial pressure - mean right atrial pressure)/cardiac output; pulmonary vascular resistance $=($ mean pulmonary artery pressure - mean pulmonary wedged pressure)/cardiac output; stroke index = cardiac index/heart rate; stroke work index (Grossman, 1974) $=$ (systolic arterial pressure pulmonary wedged pressure) $\times$ stroke index $\times$ 0.0136; minute work index (Grossman, 1974) = (stroke work index $\times$ heart rate) $/ 1000$.

Two sets of baseline observations were made at -10 and $0 \mathrm{~min}$. After the second set, pinacidil $0.2 \mathrm{mg} \mathrm{kg}^{-1}$ (range $12-19 \mathrm{mg}$ ) in $20 \mathrm{ml}$ of $0.9 \%$ saline was injected over $8 \mathrm{~min}$ through the flowguided catheter into the pulmonary artery, using a syringe pump. Observations were repeated at $10,20,30,40,55$ and 70 min after commencing infusion. The catheters were then removed and the patients returned to the ward for $24 \mathrm{~h}$ supine rest.

Results are expressed as the mean \pm s.e. mean. The values observed after drug treatment were compared with the second pre-treatment values, and the statistical significance of the differences calculated using Wilcoxon's signed rank test for matched pairs. A $P$ value of less than 0.05 was taken as an indication of significance.

\section{Results}

All subjects had normal left ventricular function (see Table 1) except for Patient 6, in whom the ejection fraction was reduced to $43 \%$ and left ventricular end diastolic pressure was $13 \mathrm{mmHg}$. Mean right atrial pressure was $0.0 \pm 0.5 \mathrm{mmHg}$. The measured haemodynamic parameters are shown in Table 2. The baseline observations at - 10 and 0 min were not significantly different and all values were within the normal range. Comparisons were therefore made with the second baseline values only.

Table 1 Clinical details of patients studied: sex, age (years), angiographic appearances of the coronary arteries

\begin{tabular}{|c|c|c|c|c|c|c|c|}
\hline Number & Sex & Age (years) & Angio & Height $(\mathrm{cm})$ & Weight (kg) & $L V E D P(\mathrm{mmHg})$ & $E F(\%)$ \\
\hline 1 & $\mathbf{M}$ & 51 & 2 & 175 & 77.0 & 2 & 71 \\
\hline 2 & $\mathbf{M}$ & 58 & 0 & 184 & 76.0 & 5 & 77 \\
\hline 3 & $\mathbf{M}$ & 42 & 0 & 167 & 82.0 & 8 & 76 \\
\hline 4 & $\mathbf{M}$ & 48 & 1 & 162 & 76.8 & 5 & 55 \\
\hline 5 & $\mathbf{F}$ & 42 & 0 & 160 & 73.0 & 1 & 87 \\
\hline 6 & $\mathbf{M}$ & 50 & 2 & 178 & 85.0 & 13 & 43 \\
\hline 7 & $\mathbf{M}$ & 41 & 0 & 167 & 69.0 & 6 & 64 \\
\hline 8 & $\mathbf{M}$ & 46 & $0^{*}$ & 177 & 95.0 & 5 & 59 \\
\hline 9 & $\mathbf{M}$ & 49 & 3 & 180 & 83.0 & 6 & 69 \\
\hline 10 & $\mathbf{F}$ & 40 & 0 & 164 & 58.4 & 0 & 74 \\
\hline \multicolumn{2}{|c|}{ (mean + s.e. mean) } & $46.7 \pm 1.8$ & & $171.4 \pm 2.7$ & $77.5 \pm 3.1$ & $5.1 \pm 1.2$ & $67.5 \pm 4.0$ \\
\hline
\end{tabular}

(Angio; $0=$ normal, $1=$ single, $2=$ double, $3=$ triple vessel disease; ${ }^{*}=$ post-angioplasty), basal left ventricular end diastolic pressure (LVEDP, $\mathrm{mmHg}$ ) and ejection fraction (EF, \%) 


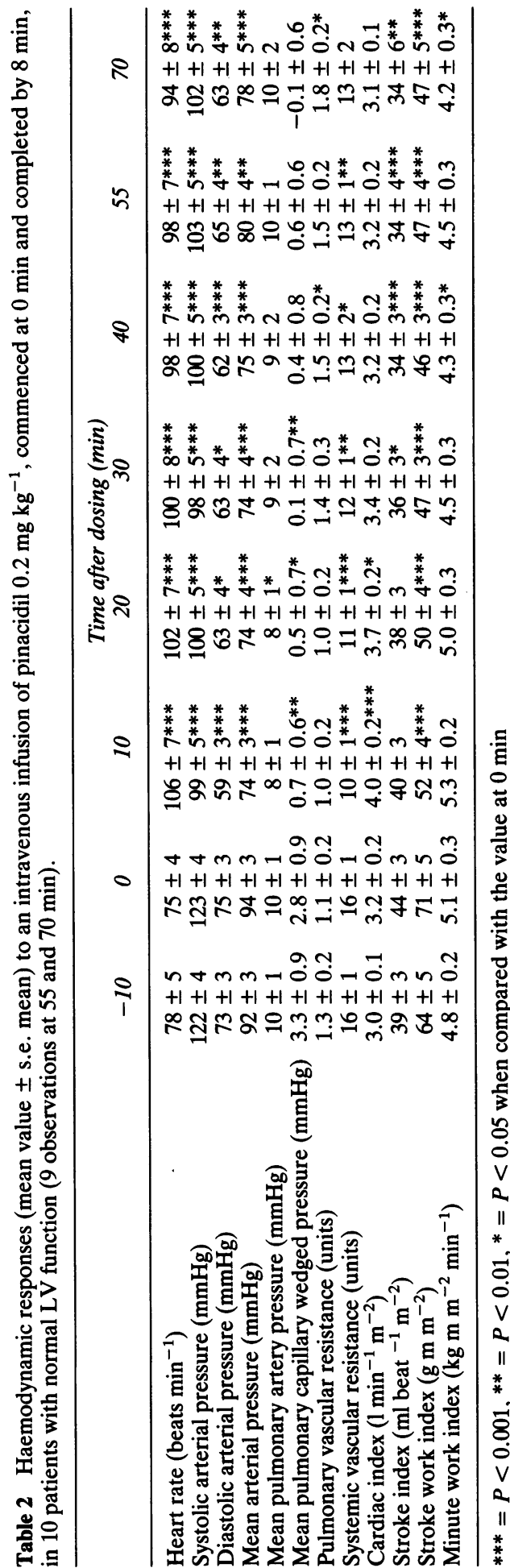

The changes in heart rate and systemic arterial pressure produced by pinacidil are shown in Table 2 and summarised in Figure 1. Heart rate was increased by over 30 beats $\mathrm{min}^{-1}$ after the end of pinacidil infusion $(10 \mathrm{~min})$. The tachycardia persisted throughout the observation period, but was less at 70 min after the commencement of infusion. Similarly, systolic, diastolic and mean systemic arterial pressures were reduced by the end of the infusion, and remained reduced for the rest of the observation period. A reduction of mean pulmonary artery pressure was observed at 20 min after commencing infusion $(P<0.05)$. Mean pulmonary capillary wedge pressure was reduced throughout the observation period, and this reduction was significant at $10(P<$ $0.01), 20(P<0.05)$ and $30(P<0.01) \mathrm{min}$ after commencing infusion.

A small increase in pulmonary vascular resistance was observed, which was significant $(P<$ 0.05 ) at 40 and $70 \mathrm{~min}$ after drug administration. The changes in cardiac index and systemic vascular resistance produced by pinacidil are shown in Table 2 and summarised in Figure 2. Cardiac index was increased after infusion, but had returned to pretreatment values by $30 \mathrm{~min}$ after commencing infusion. Similarly, systemic vascular resistance was initially reduced, but increased again during the observation period.

Stroke index was initially unchanged, but significant reductions were observed from $30 \mathrm{~min}$ after commencing drug infusion. Stroke work index was significantly reduced $(P<0.001)$ at all time intervals after administration of pinacidil. Minute work index was initially unchanged but later also reduced.

\section{Adverse effects}

Six patients reported no symptoms during the study. One patient (No. 5) had nausea and vomiting $10 \mathrm{~min}$ after completion of the study. One patient (No. 10) was aware of palpitations 10 min after commencing infusion (heart rate 126 beats $\min ^{-1}$ ). Two patients (Nos. 4 and 9) had pallor and nausea associated with progressive bradycardia and hypotension, which responded to intravenous atropine and head-down tilt. In patient No. 4 this occurred 45 min after commencing infusion, and the measurements at $\mathbf{5 5}$ and $70 \mathrm{~min}$ were not obtained. In patient No. 9, this occurred $1 \mathrm{~h}$ after completion of the study.

\section{Discussion}

Contrast agents used during coronary angiography are vasoactive, but the effects of nonionic 


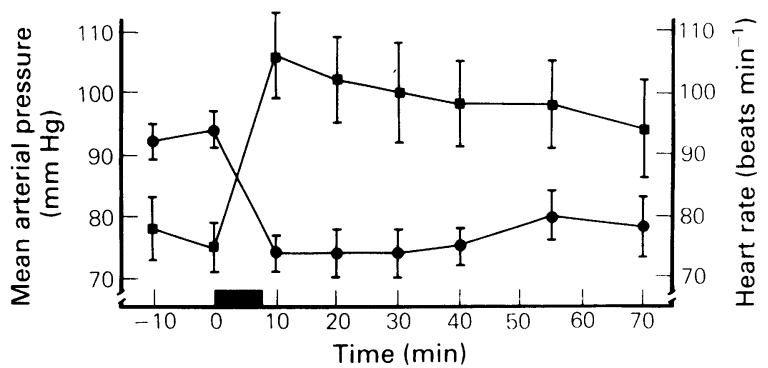

Figure 1 Effect of intravenous infusion of pinacidil $0.2 \mathrm{mg} \mathrm{kg}^{-1}$ on mean systemic arterial pressure $(\mathrm{mmHg} ; \bullet)$ and heart rate (beats $\left.\min ^{-1} ; \boldsymbol{\sigma}\right)$. The solid bar indicates the duration of infusion. Means \pm s.e. mean are indicated $(n=10$, except at 55 and $70 \mathrm{~min}$, when $n=9$ ).

media such as iopamidol are less than those observed after the earlier ionic agents (Partridge et al., 1981; Morris et al., 1985). The cardiovascular effects of iopamidol are only observed within $1 \mathrm{~min}$ of injection, and the effects of vasodilator drugs such as nifedipine and diltiazem are not potentiated (Morris et al., 1985). In the present study, the patients were haemodynamically stable prior to pinacidil infusion and so it is unlikely that iopamidol contributed to the observed responses. However, it is not possible to prove that all the observed responses were due to pinacidil as no random-order placebo infusion was given. Nevertheless, we consider it most unlikely that the marked changes observed could have occurred due to chance alone.

Pinacidil reduced systemic arterial pressure and vascular resistance, and increased heart rate and cardiac index. The effects on arterial pressure and heart rate were observed during the infusion, so that the greatest changes were seen $10 \mathrm{~min}$ after commencing infusion, and the changes continued for at least $70 \mathrm{~min}$. The effects of pinacidil on cardiac index and systemic vascular resistance were also observed $10 \mathrm{~min}$ after commencing infusion, but then declined so that by the end of observation, values had returned towards pre-treatment levels. Only small changes in pulmonary pressures were observed. The stroke index was reduced from $30 \mathrm{~min}$ after commencing infusion, which may reflect volume depletion (see below). The stroke work index was reduced at all time intervals, but this could be explained by the reduced arterial pressure; a negative inotropic effect of pinacidil is unlikely as the pulmonary wedged pressures reduced rather than increased. This hac modynamic profile suggests that pinacidil acts as a vasodilator, with an arterial rather than a venous site of action. The reduced stroke work and minute work indices would suggest that the tachycardia produced did not negate the effects of left ventricular afterload reduction.

Other studies have shown similar haemodynamic effects of pinacidil. In 15 normal subjects given pinacidil $0.2 \mathrm{mg} \mathrm{kg}^{-1}$ over $4 \mathrm{~min}$, mean arterial pressure was reduced by $16 \mathrm{mmHg}$ and heart rate increased by 24 beats $\mathrm{min}^{-1}$ within $5 \mathrm{~min}$; mean arterial pressure returned to pretreatment values in $120 \mathrm{~min}$, and heart rate in $180 \mathrm{~min}$ (Ward et al., 1984). In 12 hypertensive patients, a single oral dose of pinacidil $25 \mathrm{mg}$ reduced supine mean arterial pressure by $38 \mathrm{mmHg}$ and increased heart rate by 23 beats $\mathrm{min}^{-1}$ (Kardel et al., 1981). In another study on hypertensive patients (Carlsen et al., 1981), similar changes in arterial pressure and heart rate were observed, in addition to increases in stroke volume and

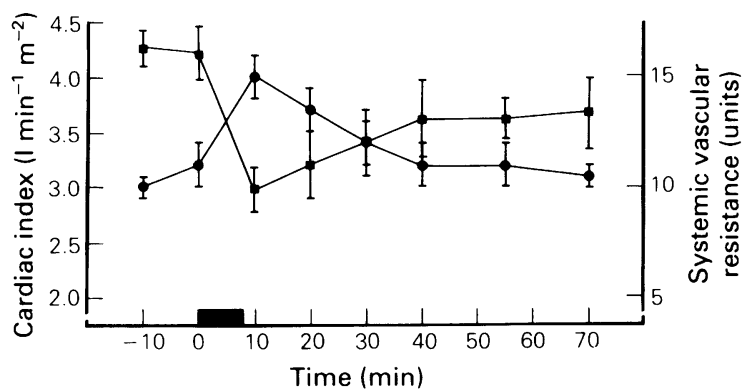

Figure 2 Effect of intravenous infusion of pinacidil $0.2 \mathrm{mg} \mathrm{kg}^{-1}$ on cardiac index $\left(1 \mathrm{~min}^{-1} \mathrm{~m}^{-2} ; \bullet\right)$ and systemic vascular resistance (units; $\boldsymbol{a})$. The solid bar indicates the duration of infusion. Means \pm s.e. mean are indicated $(n=10$, except at 55 and $70 \mathrm{~min}$, when $n=9)$. 
cardiac output (impedance cardiography) and forearm blood flow (venous occlusion plethysmography). Systemic and forearm vascular resistance were reduced. In patients with chronic congestive cardiac failure, an invasive haemodynamic study showed that pinacidil $0.2 \mathrm{mg} \mathrm{kg}^{-1}$ given intravenously reduced mean arterial pressure by $10 \mathrm{mmHg}$ and increased heart rate by 10 beats $\min ^{-1}$ (Walsh et al., 1984). Cardiac index increased, systemic vascular resistance fell and pulmonary pressures were unchanged. The present study confirms that these haemodynamic changes also occur in normotensive man.

In two patients bradycardia and marked hypotension were observed. There were no features which set these patients apart from the others studied, and cardiac function in both cases was initially normal, with a mean pulmonary wedged pressure of $5 \mathrm{mmHg}$. All patients were fasted prior to study, and this may account for the low initial left ventricular filling pressures and the marked changes in heart rate and arterial pressure observed after drug administration. In the two patients with near-syncope, mean pulmonary wedged pressure fell during the study, and so the reduction of cardiac output was unlikely to have been due to a direct negative inotropic effect of pinacidil. The prompt response to atropine, headdown tilt and fluid repletion would suggest that

\section{References}

Arrigoni-Martelli, E., Nielsen, C. K., Olsen, U. B. \& Petersen, H. J. (1980). N"-cyano-N-4-pyridyl$\mathrm{N}^{\prime}-1,2,2$,-trimethylpropylguanidine monohydrate (P1134): a new, potent vasodilator. Experientia, 36, 445-447.

Braunwald, E. (1977). Vasodilator therapy-a physio logic approach to the treatment of heart failure. New Engl. J. Med., 297, 331-333.

Carlsen, J. E., Kardel, T., Hilden, T., Tang $\varnothing$, M. \& Trap-Jensen, J. (1981). Immediate central and peripheral haemodynamic effects of a new vasodilating agent pinacidil (P1134) in hypertensive man. Clin. Physiol., 1, 375-384.

Freis, E. D., Rose, J. C., Higgins, T. F., Finnerty, F. A., Kelley, R. T. \& Partenope, E. A. (1953). The hemodynamic effects of hypotensive drugs in man. IV. 1-hydrazinophthalazine. Circulation, 8 , 199-204.

Grossman, W. (1974). In Cardiac catheterisation and angiography. Philadelphia: Lea and Febiger.

Kardel, T., Hilden, T., Carlsen, J. \& Trap-Jensen, J. (1981). N"-cyano-N-4-pyridyl-N'-1,2,2,-trimethylpropylguanidine, a new vasodilating agent: acute effect on blood pressure and pharmacokinetics in hypertensive patients. J. cardiovasc. Pharmac., 3, 1002-1007.

Koch-Weser, J. (1976). Drug therapy: hydralazine. New Engl. J. Med., 295, 320-323.

Mikkelsen, E. \& Pedersen, O. L. (1982). Comparison of the effects of a new vasodilator pinacidil and the cause was a vagal response to venous pooling.

The profile of action of pinacidil is similar to that observed after other non-specific arteriolar vasodilators such as hydralazine (Freis et al., 1953; Koch-Weser, 1976). The vasodilation produced by pinacidil results in a reflex tachycardia and increase in cardiac output, which may limit its use in the treatment of arterial hypertension when given alone. If the reflex tachycardia is reduced by concurrent administration of a $\beta$ adrenoceptor antagonist (Nicholls et al., 1986), then combined treatment may be more effective than pinacidil alone. The other main indication for after-load reduction is in the treatment of low output cardiac failure (Braunwald, 1977) and initial studies in patients with heart failure have shown a favourable haemodynamic response (Walsh et al., 1984). It is probable therefore that pinacidil will prove to be of value in the treatment both in hypertension and of low output cardiac failure.

We are grateful to our Physiological Measurement Technicians, Mrs V. Anderson, Miss B. Campbell, Mr T. Gage, Miss G. McParland and Mrs S. Wylie for their help. We are also grateful to Drs R. Burnett and $\mathrm{K}$. Willan of Leo Laboratories for supplies of pinacidil and for financial assistance.

nifedipine on isolated blood vessels. Acta Pharmac. Tox., 51, 407-412.

Morris, D. L., Wisneski, J. J. A., Gertz, E. W., Wexman, M., Axelrod, R. \& Langberg, J. (1985). Potentiation by nifedipine and diltiazem of the hypotensive response after contrast angiography. J. Am. Coll. Cardiol., 6, 785-791.

Nicholls, D. P., O'Keeffe, D. B., Morton, P., Murtagh, J. G., Scott, M.E. \& Shanks, R. G. (1984). Cardiovascular effects of pinacidil in man. Irish J. med. Sci. 153, 80-81.

Nicholls, D. P., McNeill, J., Harron, D. W. G. \& Shanks, R. G. (1986). Cardiovascular effects of pinacidil and propranolol alone and in combination in normal humans. J. cardiovasc. Pharmac., 8, 51-54.

Nielsen, C. K. \& Arrigoni-Martelli, E. (1981). Effect of a new vasodilator, pinacidil (P1134), on potassium, noradrenaline and serotonin induced contractions in rabbit vascular tissues. Acta Pharmac. Tox., 49, 427-431.

Olsen, U. B. \& Arrigoni-Martelli, E. (1983). Vascular effects in dogs of pinacidil (P1134), a novel vasoactive antihypertensive agent. Eur. J. Pharmac., 88, 389-392.

Partridge, J. B., Robinson, P. J., Turnbull, C. M., Stoker, J. B., Boyle, R. M. \& Morrison, G. W. (1981). Clinical cardiovascular experiences with iopamidol: a new non-ionic contrast medium. Clin. Radiol., 32, 451-455. 
Walsh, M. J., Barbir, M., MacMahon, B., Kelly, P. \& Branagan, J. P. (1984). Haemodynamic effects of intravenous pinacidil in chronic congestive heart failure. Irish J. med. Sci., 153, 79.

Ward, J. W. (1984). Pinacidil monotherapy for hypertension. Br. J. clin. Pharmac., 18, 223-225.
Ward, J. W., McBurney, A., Farrow, P. R. \& Sharp, P. (1984). Pharmacokinetics and hypotensive effect in healthy volunteers of pinacidil, a new potent vasodilator. Eur. J. clin. Pharmac., 26, 603-608. 